\section{PATIENT SAFETY}

\title{
Reducing Patient Radiation Dose during CT-Guided Procedures: Demonstration in Spinal Injections for Pain
}

\author{
T.M. Shepherd \\ C.P. Hess \\ C.T. Chin \\ R. Gould \\ W.P. Dillon
}

BACKGROUND AND PURPOSE: CT guidance may improve precision for diagnostic and therapeutic
spinal injections, but it can increase patient radiation dose. This study examined the impact of reducing
tube current on patient radiation exposure and the technical success for these procedures, by using
axial acquisitions for short scan lengths and eliminating nonessential imaging. MATERIALS AND METHODS: Our institutional review board approved retrospective analysis of records
from 100 consecutive outpatients undergoing spinal injections for pain before and after the CT protocol modification to reduce radiation dose. Data collected included patient age and sex, response to injection, number of sites and spinal levels treated, injection type, performing physician, CT acquisition method, number of imaging series, tube current, scan length, and DLP.

RESULTS: Image contrast was reduced with the low-dose protocol, but this did not affect technical success or immediate pain relief. Mean DLP for all procedures decreased from $1458 \pm 1022$ to $199 \pm 101$ $\mathrm{mGy} \cdot \mathrm{cm}(P<.001)$. The range of radiologist-dependent DLP per procedure also was reduced significantly with the modified protocol. Selective nerve root blocks, lumbar injections, multiple injection sites, and the lack of prior imaging were each associated with a slightly higher DLP $(<50 \mathrm{mGy} \cdot \mathrm{cm})$.

concLUSIONS: Radiation to patients undergoing CT-guided spinal injections can be decreased significantly without affecting outcome by reducing tube current, using axial acquisitions for short scan lengths, and eliminating nonessential imaging guidance. These measures also decrease variability in radiation doses between different practitioners and should be useful for other CT-guided procedures in radiology.

ABBREVIATIONS: ANOVA = analysis of variance; $\mathrm{CTDI}_{\text {vol }}=$ volume $\mathrm{CT}$ dose index; $\mathrm{DLP}=$ doselength product; $N A=$ not applicable
C T image guidance ensures accurate needle placement to localize and/or treat spinal pain, ${ }^{1}$ a common medical problem with substantial costs to society. ${ }^{2}$ In general, CT has an increasing role in interventional radiology. However, there is mounting concern in the medical community ${ }^{3}$ and general public ${ }^{4}$ regarding the potential harmful effects of medical radiation exposure. Furthermore, patient radiation doses have shown surprising variability even for the same CT protocol at different institutions. ${ }^{5}$ Recent editorials have advocated for the development and widespread adoption of more uniform CT acquisitions to reduce both radiation dose and variability. ${ }^{3,6}$

A number of different strategies have been proposed to reduce $\mathrm{CT}$ radiation dose with varying effects on resultant

Received November 24, 2010; accepted after revision February 23, 2011.

From the Neuroradiology Division, Department of Radiology and Biomedical Imaging, University of California, San Francisco, San Francisco, California

T.M.S. was funded through National Institutes of Health/National Institute of Biomedical Imaging and Bioengineering T32 EB001631-05. C.P.H. is funded by the GE Radiology Research Academic Fellowship program.

Paper previously presented at: Annual Meeting of the Western Neuroradiological Society, September 28 to October 2, 2010; Wailea, Hawaii.

Please address correspondence to Christopher P. Hess, MD, PhD, Neuroradiology, Department of Radiology and Biomedical Imaging, Box 0628, University of California, San Francisco, 505 Parnassus Ave, L-358, San Francisco, CA 94143-0628; e-mail: christopher. hess@ucsf.edu

Indicates open access to non-subscribers at www.ajnr.org

http://dx.doi.org/10.3174/ajnr.A2634 image quality. ${ }^{7,8}$ Tailoring the acquisition protocol to the diagnostic or interventional goal of the procedure is 1 straightforward approach. ${ }^{9}$ In the case of spinal injections, 3 observations can be used to design an appropriate protocol for CT guidance. First, when the site of injection is known a priori, it should be possible to target imaging only to the structures of interest. Second, osseous spine anatomy has high inherent contrast such that $\mathrm{x}$-ray tube current throughout the imaging procedure might be reduced. Doses for lumbar CTs in adult patients with suspected disk herniation were reduced 35\% in this manner without degrading image quality, ${ }^{10}$ and experiments in phantoms have predicted substantial dose reductions with lower tube currents during lumbar spine injections. ${ }^{11}$ Finally, because CT-guided spinal injections require imaging only short lengths along the scanner axis, switching from helical to axial acquisition modes also can reduce the radiation dose from over-ranging. ${ }^{12-14}$

This study describes the impact of several steps that were taken in our academic practice to reduce patient radiation during CT-guided injection procedures for spinal pain. Specifically, we implemented a protocol by using anatomically targeted survey imaging, lower tube current, and axial acquisitions for short image lengths for all CT-guided spinal injections. We found that these 3 steps achieved substantial reductions in both the radiation to patients and in the variability in patient radiation for procedures performed by different radiologists. 


\section{Materials and Methods}

\section{Study Design}

Our institutional review board approved this study. In March 2010, our neuroradiology section made several modifications to CT protocols for spine interventions to reduce the radiation doses to our patients. Specifically, tube-current and scanning lengths were reduced, and acquisitions were changed from helical to axial for most imaging series. To assess the impact of these measures on radiation reduction, we retrospectively collected data for the first 50 consecutive patients that followed the implementation of these changes. For comparison, we also collected data retrospectively for 50 consecutive patients undergoing procedures in March 2009, 1 year preceding the implementation of the dose-reduction steps.

\section{Patient Selection}

All patients undergoing CT-guided epidural, facet joint, nerve root, or medial branch blocks to relieve spinal pain were included. Patients undergoing other CT-guided spinal interventions such as percutaneous biopsy, kyphoplasty, vertebroplasty, and radio-frequency ablation were excluded. Only the first procedure for a patient undergoing multiple procedures in the same year was included (to limit the impact of specific individual factors such as body habitus). Patients also were excluded if the dose report was not available.

\section{Spinal Injections and CT Technique}

All spinal injection procedures were performed on the same 64-section CT scanner (LightSpeed VCT; GE Healthcare, Milwaukee, Wisconsin) at a single outpatient facility (rotation time, 0.8 seconds; tube potential, $120 \mathrm{kV}$; section thickness, $2.5 \mathrm{~mm}$; collimation, $40 \mathrm{~cm}$; and helical pitch and detector coverage, 1.375 and $40 \mathrm{~mm}$, respectively). The noise index was not adjusted. Individual supervising neuroradiologists altered only tube current, scan length, and the CT acquisition method.

\section{Data Collection}

Data were collected using the departmental PACS system by reviewing CT images, dose reports, and interpretative reports. Each series of images from a particular study was assigned to 1 of 3 phases: a "survey" phase, consisting of an initial CT acquisition by using a fiducial grid for procedure planning; a "guide" phase, in which multiple image series were acquired as $\geq 1$ needle was advanced to specific anatomic targets for injection; and a "contrast" phase, in which CT was performed to verify correct needle placement following injection of iodinated contrast. The presence or absence of metallic hardware within the region of interest also was recorded because a higher tube current may have been used in these cases to better visualize regional anatomy. The difference between time stamps on the first and last images was used as a surrogate for the total procedural time.

Information collected from the radiology interpretative report included patient sex and age, the supervising attending neuroradiologist (the same 3 neuroradiologists supervised procedures in both years), spinal anatomy treated, number of sites injected, injection type (epidural, facet joint, selective nerve root, or medial branch block), pre- and postprocedure pain assessment, and the dates of available prior cross-sectional imaging for the spinal anatomy being treated. The spinal anatomy was recorded as cervical, thoracic, lumbar or sacral; and transitions were assigned to the superior level (eg, a C7-T1 injection was cervical). Spinal levels were divided by intervertebral disk spaces per standard convention, and each needle used during the procedure was considered a single injection site (eg, an injection of both articulating facets at L4-L5 was recorded as 1 level and 2 sites). Patient-reported pain scales immediately before and after the procedure (rated $0-10$, with 0 being no pain) were recorded in the $64 / 100$ patients for whom this information was documented in the radiology report.

The dose report provided a separate acquisition method (helical or axial), scan length in centimeters, tube current in milliamperes, and DLP in milligrays per'centimeter for each imaging series. The scan length for an individual scan series was measured as the sum of all imaged regions along the $\mathrm{z}$-axis without including gaps in cases in which more than a single level was injected. The dose report also provided the total examination DLP. All data were entered into a password-protected database on an encrypted computer. Statistical comparisons used unpaired Student $t$ tests or 1-way ANOVAs with post hoc Tukey tests for significance when differences were found.

\section{Radiation Dose Estimation}

This study focused on quantifying reductions to the DLP during CTguided spinal injections. DLP is the product of scan length and the $\mathrm{CTDI}_{\mathrm{vol}}$. The CTDI $\mathrm{vol}_{\text {al }}$ and DLP are precisely defined, reproducible, and conform to industry and regulatory standards. ${ }^{15-18}$ The DLP (milligrays per centimeter) is often used to estimate the effective dose (millisievert) in adults of standard physique by multiplying the DLP by $0.0059,0.014$, or $0.015 \mathrm{mSv} / \mathrm{mGy} \cdot \mathrm{cm}$ for the cervical, thoracic, or lumbosacral spine, respectively. ${ }^{19}$ The accuracy of the estimated effective doses, however, depends on individual patient factors (eg, body habitus). Hence, a reduction in DLP will still correspond to a reduction in a patient's effective dose, but the estimated magnitude of the latter reduction is not as precise.

\section{Results}

Images obtained during the different procedural phases in 2010 showed reduced perceived contrast compared with procedural images obtained in 2009, which was attributed to the lower tube current (Fig 1). However, there remained sufficient detail for identifying relevant vertebral anatomy and the positions of fiducials, needles, and iodinated contrast throughout the procedure in all cases. All 100 cases were technically successful for injection of the a priori anatomic target without immediate postprocedural complications. Immediate reduction in patient-reported pain also did not differ between years (Table 1), with $92 \%$ of patients reporting at least mild reduction in pain scores.

In 2010, changing most CT acquisitions from helical to axial, reducing tube current, and reducing nonessential imaging (Table 2) lowered the mean total DLP for the procedure by $86 \%$ from $1458 \pm 1022$ to $199 \pm 101 \mathrm{mGy} \cdot \mathrm{cm}(P<.001)$. The DLP for each phase of the spinal injection procedure also was reduced (Fig $2 A$ ). More than $70 \%$ of the total DLP reduction was due to lower mean DLP during the guide phase of the procedure (reduced by $926 \mathrm{mGy} \cdot \mathrm{cm}$ in 2010). Per image series, the DLP during the guide phase was reduced by $>95 \%$ from $86 \pm 51$ to $3 \pm 3 \mathrm{mGy} \cdot \mathrm{cm}$ from 2009 to $2010(P<.001)$. This corresponded to an $82 \%$ reduction in minimum tube current for an individual image series during the guide phase $(P<.001)$ (Table 2). Furthermore, the number of imaging series required to advance the needle to the target decreased from $12.3 \pm 5.8$ in 2009 to $9.7 \pm 5.4$ in $2010(P=.022)$. Overall, the extent to which different phases of the examina- 


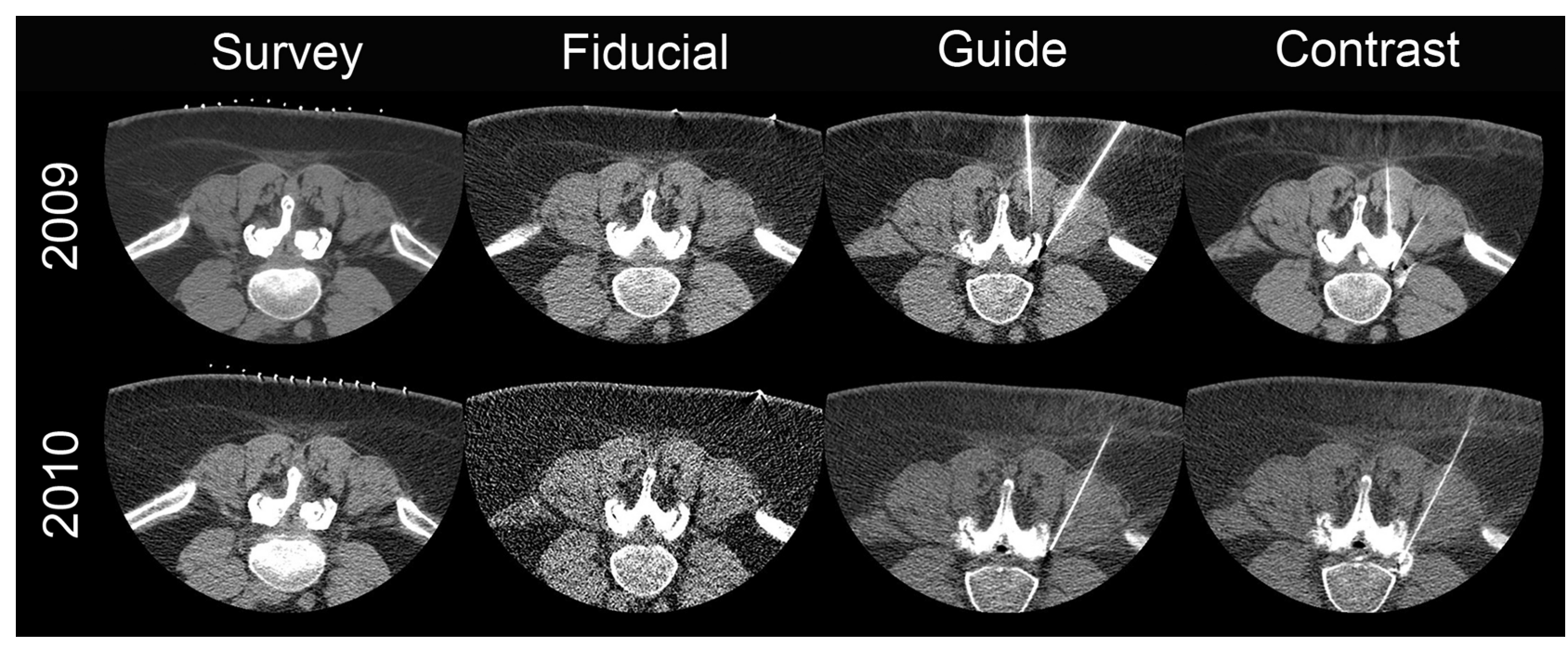

Fig 1. Comparison images for the same patient who received a left $L 4-5$ facet block, a left $L 4-5$ synovial cyst fenestration, and a left $L 4$ selective nerve root block in both 2009 and 2010 . All acquisitions in 2009 were helical, whereas in 2010, only the initial survey-phase CT images were acquired helically. The tube current was reduced from 549 to 149 mA in the survey image, from 84 to $30 \mathrm{~mA}$ in the fiducial bead image, from 84 to $50 \mathrm{~mA}$ in the guide phases of the study, and from 199 to $50 \mathrm{~mA}$ in the postcontrast images. Total examination DLP in 2010 was reduced $91 \%$.

Table 1: Group comparisons between patients receiving CT-guided spinal injections in 2009 and $2010^{\mathrm{a}}$

\begin{tabular}{lccr}
\hline Patient Group & 2009 & 2010 & $\begin{array}{r}T \text { Test } \\
P \text { Value }\end{array}$ \\
\hline Age (yr) & $55.6 \pm 14.0$ & $59.9 \pm 14.0$ & .128 \\
Male & $42 \%(21)$ & $58 \%(29)$ & NA \\
Injection sites & $2.0 \pm 1.0(100)$ & $2.1 \pm 1.3(103)$ & .667 \\
$\quad$ Medial branch blocks & $0.1 \pm 0.3(4)$ & $0.1 \pm 0.4(4)$ & 1.000 \\
$\quad$ Facet joint blocks & $0.4 \pm 0.8(22)$ & $0.6 \pm 1.3(28)$ & .357 \\
$\quad$ Selective root blocks & $1.3 \pm 0.9(63)$ & $1.0 \pm 1.0(49)$ & .118 \\
$\quad$ Epidural blocks & $0.2 \pm 0.4(11)$ & $0.4 \pm 0.6(22)$ & .053 \\
Injection levels & $1.5 \pm 0.7(77)$ & $1.6 \pm 1.1(81)$ & .589 \\
$\quad$ Cervical levels & $0.7 \pm 0.8(35)$ & $0.2 \pm 0.6(12)$ & .001 \\
$\quad$ Thoracic levels & $0.1 \pm 0.5(5)$ & $0.1 \pm 0.3(4)$ & 1.000 \\
$\quad$ Lumbar levels & $0.6 \pm 0.8(30)$ & $1.1 \pm 1.2(53)$ & .016 \\
$\quad$ Sacral levels & $0.1 \pm 0.4(7)$ & $0.2 \pm 0.6(12)$ & .329 \\
Hardware present & $24 \%(12)$ & $14 \%(7)$ & NA \\
Reported pain & $6.2 \pm 1.8(28)$ & $6.3 \pm 2.3(36)$ & .809 \\
Immediate pain decrease & $3.9 \pm 2.4$ & $4.4 \pm 2.6$ & .320 \\
Procedure time (min) & $45.4 \pm 19.1$ & $43.1 \pm 17.8$ & .535 \\
\hline a Fifty patients per group (mean \pm SD) (total). & &
\end{tabular}

${ }^{b}$ The parentheses for this row indicate the number of patients with available data.

tion contributed to overall patient radiation changed (Fig 3) so that in 2010 the survey phase of the examination now contributed the largest component to the total patient effective dose (75\%) for the procedure.

Most patients (79\%) underwent cervical or lumbar-only CT-guided injections for neck or lower back pain, respectively. Both of these groups showed similar DLP reductions between 2009 and 2010 (Fig 2B, -C). In 2009, cervical-only procedures had a $70 \%$ higher guide-phase DLP than lumbar-only procedures $(P<.04)$, corresponding to a higher mean total examination DLP of $476 \mathrm{mGy} \cdot \mathrm{cm}(P<.0001)$. Most of the DLP difference can be attributed to use of higher tube current in cervical procedures during 2009; for example, the minimum tube current for an image series obtained during the guide phase was $175 \%$ higher $(P<.001)$ (Table 2$)$. The difference in DLP between cervical and lumbar procedures in 2009, however, was not observed in 2010. Besides the global reductions
Table 2: Group comparisons between selected CT-acquisition parameters for patients receiving CT-guided spinal injections in 2009 and $2010^{\mathrm{a}}$

\begin{tabular}{llccr}
\hline & & & & $T$ Test \\
Parameter & Anatomy & 2009 & 2010 & $P$ Value \\
\hline Minimum guide-phase & All studies & $75.0 \pm 61.2$ & $13.4 \pm 7.3$ & $<.001$ \\
tube current (mA) & Cervical only & $104.0 \pm 68.2$ & $16.7 \pm 11.2$ & $<.001$ \\
& Lumbar only & $37.7 \pm 29.0$ & $11.7 \pm 4.9$ & $<.001$ \\
Total image series & All studies & $12.3 \pm 5.8$ & $9.7 \pm 5.4$ & .022 \\
& Cervical only & $14.0 \pm 6.1$ & $10.9 \pm 4.6$ & .178 \\
& Lumbar only & $10.8 \pm 3.3$ & $9.3 \pm 4.9$ & .269 \\
Survey scan length & All studies & $100.4 \pm 36.4$ & $94.5 \pm 38.9$ & .436 \\
$(\mathrm{~mm})$ & Cervical only & $90.9 \pm 56.6$ & $56.6 \pm 20.3$ & .088 \\
& Lumbar only & $108.0 \pm 103.0$ & $103.0 \pm 40.0$ & .816 \\
\hline
\end{tabular}

a Mean \pm SD.

${ }^{b}$ Note, only $i$ series each was assigned to the survey and contrast phases of the procedure; the remainder was during the guide phase.

in tube current and DLP for all phases of the examination, there also was a $40 \%$ decrease in the scan length of the survey phase for cervical-only procedures from $90.9 \pm 30.9$ to $56.6 \pm$ $20.3 \mathrm{~mm}(P=.088)$ (Table 2).

Radiation-dose reductions were consistent for epidural, facet, or selective nerve root blocks (Fig 4). Compared with all other injection types studied, selective nerve root blocks had higher total examination DLPs in both 2009 and 2010 ( $P=$ .004). In 2010, these total DLP differences were attributed to higher tube current (159.5 versus $59.5 \mathrm{~mA}, P=.0063$ ) and higher DLP (170.4 versus $57.2 \mathrm{mGy} \cdot \mathrm{cm}, P=.0045)$ during the survey phase, as well as higher tube current ( 61 versus 18.2 $\mathrm{mA}, P=.0149$ ) during the contrast phase. There was a trend toward increased tube current during the guide phase as well $(P=.121)$. These differences were predicted because needle placement for selective nerve root blocks requires visualization of more detailed soft-tissue anatomy compared with other CT-guided spinal injections (which appears to have been accomplished with increased tube current). The DLP for other injection types studied did not differ statistically.

Radiation dose for CT-guided spinal injections showed significant reductions under the supervision of all 3 attending 


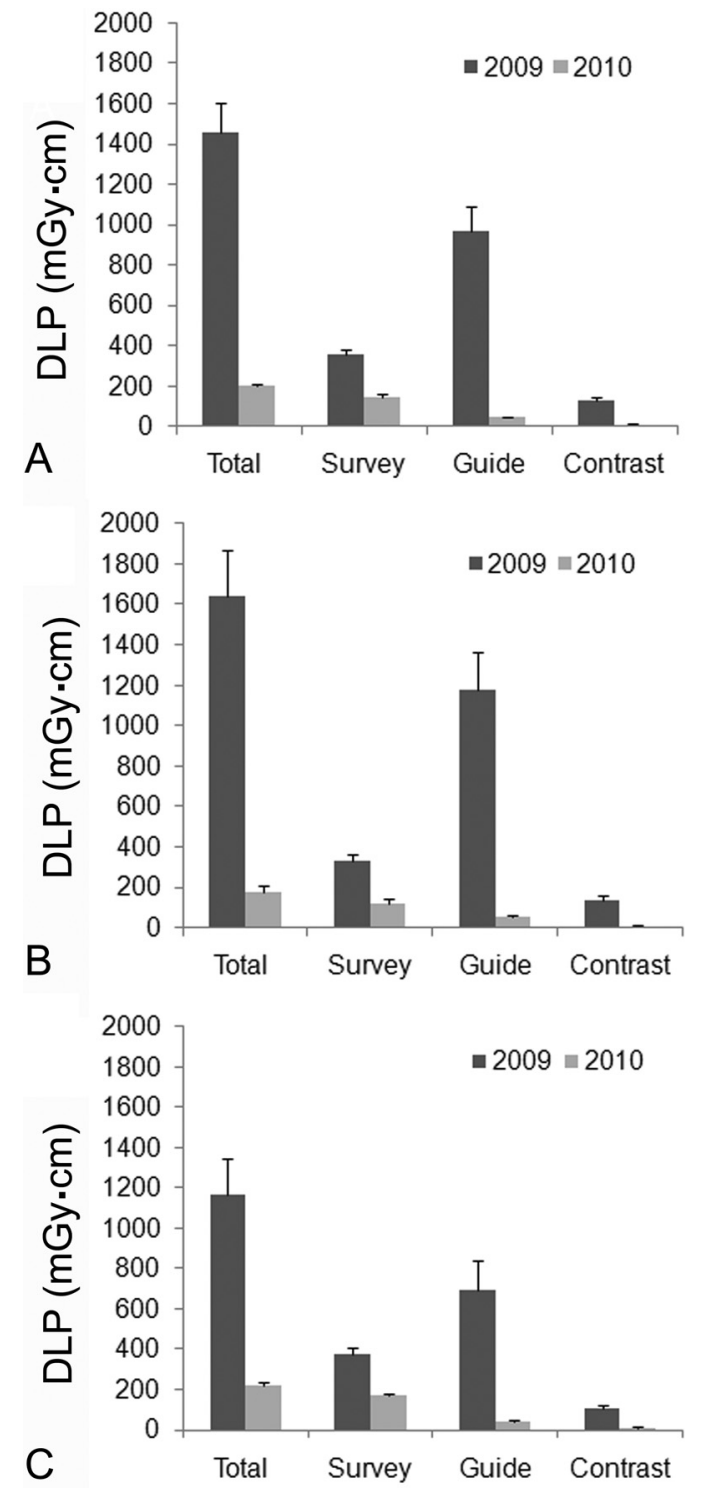

Fig 2. Between 2009 and 2010, there were significant reductions in DLP (milligrays per centimeter) for the total procedure as well as survey, guide, and contrast phases for all cases $(A)$ and cervical-only $(B)$ and lumbar-only $(C)$ CT-guided spinal injections $(P<.003$ for all individual 2009 versus 2010 comparisons) (bar graph = mean \pm standard error of the mean). The mean effective dose for cervical-only and lumbar-only CT-guided injections was reduced in 2010 from 9.7 to $1.1 \mathrm{mSv}$ and from 17.5 to $3.3 \mathrm{mSv}$, respectively. DLP for some phases of the procedure also differed significantly within the same year (see "Results").

neuroradiologists from 2009 to $2010(P<.002$, all comparisons) (Fig 5). Radiologist-dependent variability in total DLP decreased substantially as well; the largest mean DLP difference between supervising neuroradiologists decreased 95\% from $1776 \mathrm{mGy} \cdot \mathrm{cm}$ in 2009 to $94 \mathrm{mGy} \cdot \mathrm{cm}$ in 2010 .

Dose reduction persisted even when total procedure DLP was normalized to the number of spinal levels or sites injected (Fig 6). Alternatively, single- and multiple-injection-site procedures can be compared. In 36 of the 100 patients during 2009-2010, only 1 site was injected; as expected, these procedures took significantly less time (33.4 minutes versus 50.4 minutes) and supervising neuroradiologists chose shorter scan lengths for survey images (78.1 versus $108.3 \mathrm{~mm}$ ) compared with multiple-site injections (both comparisons, $P<$ $.0001)$. There also was a trend toward lower total examination
DLP, with 1-site injections in 2010 (166.2 versus 216.9 $\mathrm{mGy} \cdot \mathrm{cm}, P=.089$ ) not present in the 2009 group or combined data. The absolute difference in DLP for 2010 between single- and multiple-site injections $(50.7 \mathrm{mGy} \cdot \mathrm{cm})$ was 25 fold smaller than the overall reduction in DLP between 2009 and $2010(1259 \mathrm{mGy} \cdot \mathrm{cm})$.

Helical acquisitions were used in both years for the survey phase; however, axial replaced helical acquisitions for the guide phase of all procedures and the contrast phase of most procedures (36 of 50) in 2010. Notably, DLP was significantly higher for the 14 procedures for which helical acquisitions were still used during the contrast phase $(35.8 \pm 19.6$ versus $5.7 \pm 4.4 \mathrm{mGy} \cdot \mathrm{cm}$; ANOVA, $P<.001)$.

Prior cross-sectional imaging of the relevant spinal anatomy within the previous year was available for 36 and 39 patients in 2009 and 2010, respectively. In 2010, higher maximum tube current was used for the guide phase in patients without imaging during the previous year (58.9 \pm 38.4 versus $41.5 \pm 22.0 \mathrm{~mA}, P=.058)$. These patients also had higher mean total DLP (216.8 \pm 119.3 versus $193.5 \pm 96.4$ $\mathrm{mGy} \cdot \mathrm{cm})$, but the difference was not significant $(P=.505)$. Otherwise, the availability of prior imaging did not appear to affect procedure time or supervising neuroradiologists' other choices for the CT acquisition. Procedures in the few patients with hardware $(19 / 100)$ also trended toward increased total DLP (1118 versus $760 \mathrm{mGy} \cdot \mathrm{cm}, P=.144$ ) due to significantly increased tube current selected during the guide phase (195.5 versus $117.8 \mathrm{~mA}, P=.009)$.

\section{Discussion}

In early 2010, we attempted to aggressively reduce patient radiation during CT-guided spine injections by decreasing tube current for each phase of the procedure, switching short image length acquisitions from helical to axial, and minimizing the scan lengths and series used. Supervising neuroradiologists remained free to alter CT acquisitions on the basis of their clinical judgment, and there was no concurrent monitoring. Compared with 2009, these changes led to a dramatic $86 \%$ reduction in total DLP per CT-guided spine injection procedure in 2010 (Fig 2, $P<.001$ ).

Image contrast remained adequate for precise location of fiducials, needles, and relevant anatomy (Fig 1). Supervising neuroradiologists noted that undiluted contrast injection also reduced tube-current requirements for confirming the appropriate needle position. Although long-term outcomes were not assessed in this study, all procedures were technically successful without complications, and there were no differences between pre- and postprocedure pain scores (Table 1). In 2010, however, CT-guided spinal injections were successful with estimated effective doses as low as $0.17 \mathrm{mSv}$ (Fig 7), a dose that is equivalent or lower than has been reported for fluoroscopy-guided procedures. ${ }^{20,21}$ Mean estimated effective doses for cervical-only or lumbar-only spine injections were reduced in 2010 to 1.1 and $3.3 \mathrm{mSv}$, respectively. Radiation-dose reductions between 2009 and 2010 also were consistent for epidural, facet block, and selective nerve root blocks (Fig 4) or when normalized for the number of sites or levels injected (Fig 6).

With the short image lengths used during the procedures (generally $<10 \mathrm{~cm}$ ), particularly during the guide and contrast 
2009

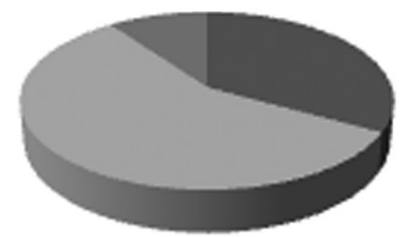

2010

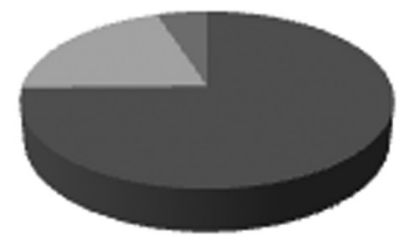

- Survey

Guide

- Contrast

Fig 3. Relative contributions from the survey, guide, and contrast phases of CT-guided spinal injections changed substantially between 2009 and 2010 . Significant reductions in DLP during the guide phase of the examination in 2010 lowered its contribution to total procedure DLP from $57 \%$ to $20 \%$. In 2010 , almost $75 \%$ of the patient dose came from the initial survey CT images obtained for identifying anatomy relevant to the patient's spinal pain and prescribing the course of needle insertion.

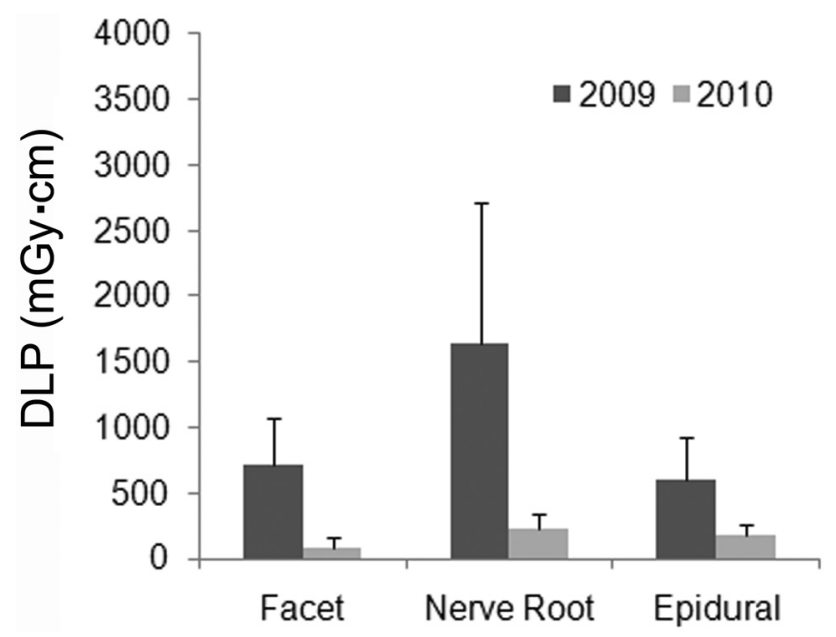

Fig 4. Comparison of total examination doses for different CT-guided procedures that involved only 1 specific type of injection (facet joint, selective nerve root, or epidural block) (bar graph $=$ mean \pm SD). Between 2009 and 2010, the total dose decreased significantly for all 3 specific types of CT-guided spinal injections (ANOVA, $P<.004$ for all comparisons). Selective nerve root block procedures were consistently higher in total dose, but this trend only reached statistical significance compared with facet joint block procedures in $2010(P=.004)$.

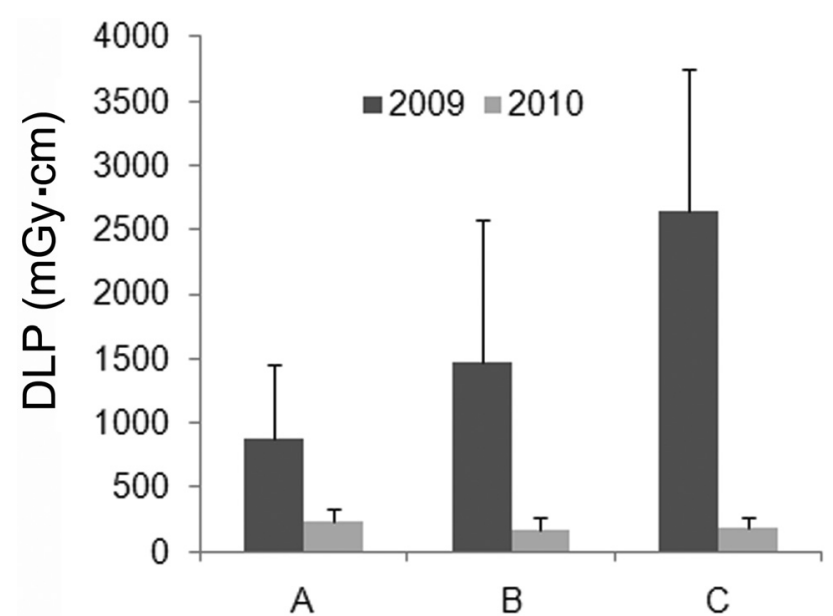

Fig 5. Dose reductions were observed for CT-guided spinal injections by individual supervising neuroradiologists (all 2009 versus 2010 comparisons, $P<.003$ ) (bar graph $=$ mean \pm SD). Comparisons between supervising neuroradiologists in the same year showed only trends toward statistically significant differences (ANOVA, $P=.167$ for either 2009 or 2010) due to the large variability in total DLP.

phases, helical acquisitions increased radiation dose from over-ranging. ${ }^{12-14}$ Hence, switching to an axial acquisition during these phases also contributed significantly to dose reductions. This was best illustrated by decreased DLP observed

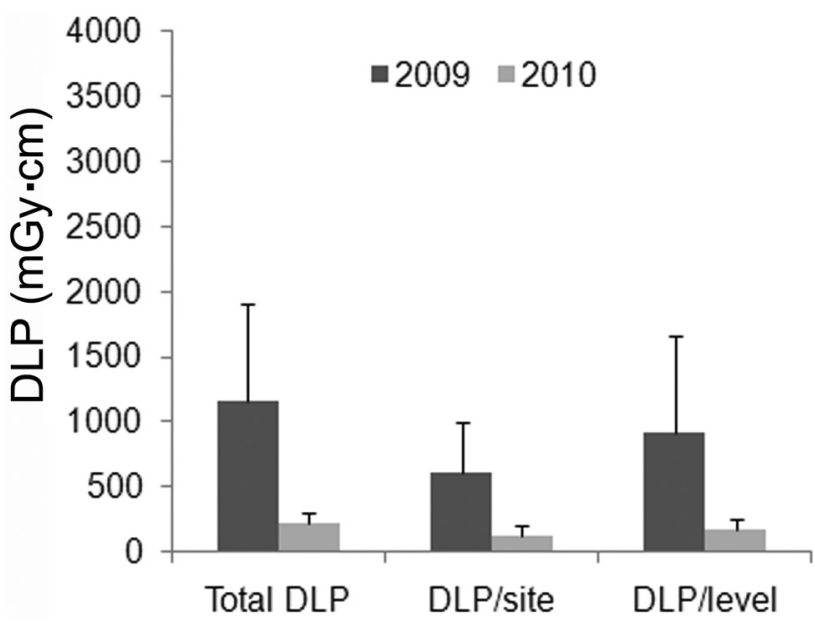

Fig 6. Lumbar-only procedures varied in the total number of sites $(2.2 \pm 1.3,1-7)$ or levels $(1.5 \pm 1.0,1-7)$ treated per encounter (mean $\pm S D$, range), but the dose reduction observed persisted even when total examination dose (total DLP) was normalized for the number of sites (DLP/site) or levels (DLP/level) injected during the procedure (bar graph $=$ mean \pm SD). All 3 ways of analyzing procedural DLP showed approximately $80 \%$ reductions between 2009 and $2010(P<.0001)$. Similar large dose reductions also persisted for cervical-only procedures when normalized for the number of sites or levels treated $(P<.002)$.

during the contrast phase of 2010 examinations when axial and helical acquisitions were compared directly. Procedure DLPs were further reduced by $21 \%$ fewer image series and $40 \%$ decreased image length for cervical-only procedures ( Table 2). Overall, the changes implemented affected the guide phase most, in which $70 \%$ of the observed total radiation reduction occurred.

This study highlighted the fact that there can be substantial radiologist-dependent variability in patient radiation during CT-guided procedures (Fig 5). Using the proposed dose-reduction strategy, differences in DLP between the highest and lowest supervising neuroradiologists were reduced 95\% from $1776 \mathrm{mGy} \cdot \mathrm{cm}$ in 2009 to $94 \mathrm{mGy} \cdot \mathrm{cm}$ in 2010 . The reduced variability in radiologist-dependent procedural radiation came without mandates or monitoring and meets recent proposals that doses for CT protocols become more consistent and independent of particular supervising neuroradiologists and/or institutions. ${ }^{3,6}$

A secondary aim of this study was to identify additional patient or procedural features that alter supervising neuroradiologists' choices of CT acquisition parameters and hence affect patient dose. Male sex, selective nerve root blocks, lumbar level injections, multiple injection sites, or absence of imaging within the previous year appeared to increase patient 

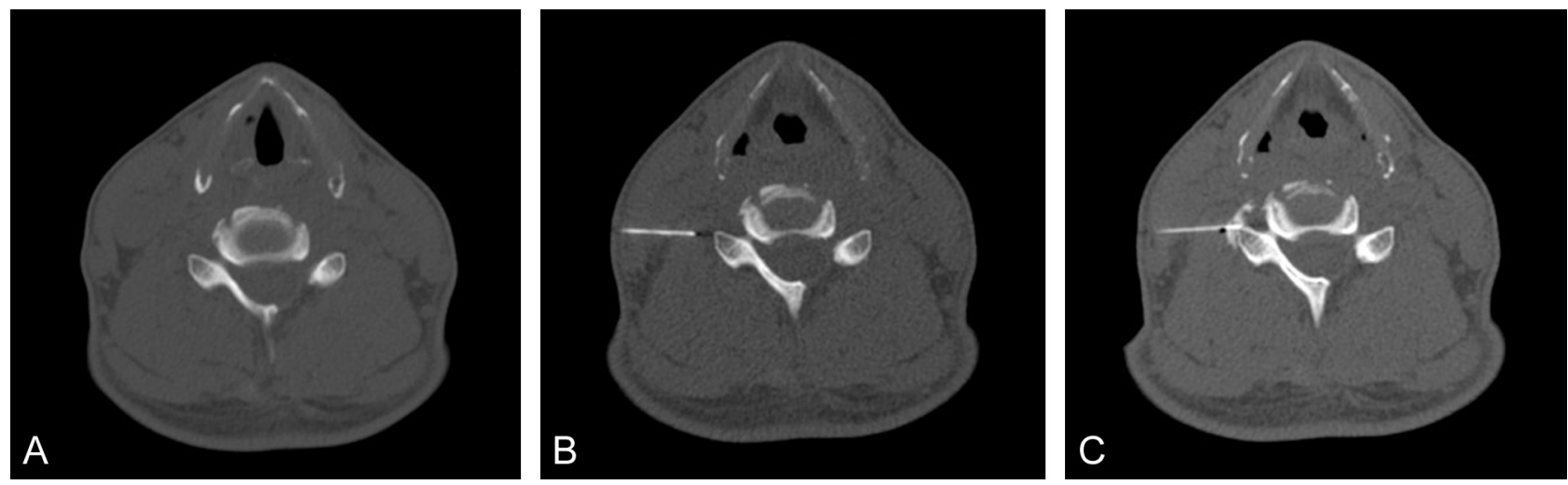

Fig 7. Sample images from a 2010 study with aggressive dose reduction during a technically successful CT-guided right C6 selective nerve root block. Only the survey CT images were acquired helically (scan length $=50 \mathrm{~mm}$ ). The milliampere/DLP (milligrays per centimeter) was 29/20.1, 10/0.5, and 20/1.0 for the survey $(A)$, guide $(B)$, and contrast $(C)$ images, respectively (note, 8 guide series with slightly varying milliamperes or ranges were obtained). Total examination DLP was $28.6 \mathrm{mGy} \cdot \mathrm{cm}$, giving an estimated total effective dose of only $0.17 \mathrm{mSv}$. This dose is comparable with or lower than that for fluoroscopy-based spinal injections for pain.

radiation. However, these factors were not additive, and the increased DLP observed for any individual factor only ranged between 23.3 and $50.7 \mathrm{mGy} \cdot \mathrm{cm}$ in 2010 (the latter only increases the effective dose by $0.7 \mathrm{mSv}$ in lumbar spine injections).

This retrospective study had some limitations. The study was not randomized, and the patient groups before and after implementing the dose-reduction strategies differed in some respects (Table 1). Specifically, in 2010, a greater proportion of patients were male and a smaller number had metallic spinal hardware. Moreover, patients in 2010 had more lumbar and fewer cervical injections than those in 2009. Furthermore, as a retrospective study, other factors that could alter the real-time choice of scan parameters such as body habitus could not be assessed. This study was designed to examine radiation dose reductions for CT-guided spinal injections and did not compare radiation doses between fluoroscopy and these injections. ${ }^{10}$ Fluoroscopy-guided spinal injections are performed by different specialists at our institution. The accurate estimation of radiation from fluoroscopy procedures also is not simple.

\section{Conclusions}

Patient radiation from CT-guided spinal procedures decreased significantly without affecting the technical success of the procedure by reducing tube current, switching to axial acquisitions for short scan lengths, and eliminating nonessential imaging. Most optimization came from decreased contributions during the guide phase (when the needle was advanced to its anatomic target). Procedural DLP showed substantial variability, depending on the supervising neuroradiologist, and was reduced by $95 \%$ after implementation of this dose-reduction strategy. The radiation reductions observed should be valid for other CT-guided procedures in radiology. Adding anatomy or attenuation-based tube-current modulation, ${ }^{22-24}$ noise-reduction algorithms, ${ }^{25}$ and/or tubevoltage reductions in nonobese patients ${ }^{26}$ may further reduce patient radiation doses. Prospective studies also may identify other patient and procedural characteristics that affect patient doses during CT-guided procedures.

\section{Acknowledgments}

The authors appreciate assistance from radiology technologists John Jang and Susan Williams.

\section{References}

1. Gangi A, Dietemann JL, Mortazavi R, et al. CT-guided interventional procedures for pain management in the lumbosacral spine. Radiographics 1998;18:621-33

2. Deyo RA, Weinstein JN. Low back pain. N Engl J Med 2001;344:363-70

3. Brenner DJ, Hall EJ. Computed tomography: an increasing source of radiation exposure. N Engl J Med 2007;357:2277-84

4. Bogdanich W. Radiation overdoses point up dangers of CT scans. New York Times. October 16, 2009;A13.

5. Smith-Bindman R, Lipson J, Marcus R, et al. Radiation dose associated with common computed tomography examinations and the associated lifetime attributable risk of cancer. Arch Intern Med 2009;169:2078-86

6. Smith-Bindman R. Is computed tomography safe? N Engl J Med 2010;363:1-4

7. Kalra MK, Maher MM, Toth TL, et al. Strategies for CT radiation dose optimization. Radiology 2004;230:619-28

8. Catalano C, Francone M, Ascarelli A, et al. Optimizing radiation dose and image quality. Eur Radiol 2007;17(suppl 6):F26-32

9. Abul-Kasim K, Overgaard A, Maly P, et al. Low-dose helical computed tomography $(\mathrm{CT})$ in the perioperative workup of adolescent idiopathic scoliosis. Eur Radiol 2009;19:610-18. Epub 2008 Sep 23

10. Bohy $\mathrm{P}$, deMaertelaer V, Roquigny A, et al. Multidetector CT in patients suspected of having lumbar disk herniation: comparison of standard-dose and simulated low-dose techniques. Radiology 2007;244:524-31

11. Schmid G, Schmitz A, Borchardt D, et al. Effective dose of CT- and fluoroscopy-guided perineural/epidural injections of the lumbar spine: a comparative study. Cardiovasc Intervent Radiol 2006;29:84-91

12. Nagel HD. CT parameters that influence the radiation dose. In: Tack D, Gevenois PA, eds. Radiation Dose from Adult and Pediatric Multidetector Computed Tomography. Berlin, Germany: Springer-Verlag; 2007:51-80

13. van der Molen AJ, Geleijns J. Overranging in multisection CT: quantification and relative contribution to dose-comparison of four 16-section CT scanners. Radiology 2007;242:208-16. Epub 2006 Nov 7

14. Tzedakis A, Damilakis J, Perisinakis K, et al. The effect of $\mathbf{z}$ overscanning on patient effective dose from multidetector helical computed tomography examinations. Med Phys 2005;32:1621-29

15. Huda W, Mettler FA. Volume CT dose index and dose-length product displayed during CT: what good are they? Radiology 2011;258:236-42

16. International Electrochemical Commission. International Standard IEC 60601-244: Part 2-44-Medical Imaging Equipment. Particular Requirements for the Basic Safety and Essential Performance of X-Ray Equipment for Computed Tomography. 3rd ed. Geneva, Switzerland: International Electrochemical Commission; 2009

17. International Electrochemical Commission. International Standard IEC 61223-2-6: Part 2-6-Evaluation and Routine Medical Testing in Medical Imaging Departments. Constancy Tests-Imaging Performance of Computer Tomography X-Ray Equipment. 2nd ed. Geneva, Switzerland: International Electrochemical Commission; 2006

18. International Electrochemical Commission. International Standard IEC 61223 3-5: Part 3-5-Evaluation and Routine Medical Testing in Medical Imaging Departments. Acceptance Tests-Imaging Performance of Computed Tomography $X$-Ray Equipment. Geneva, Switzerland: International Electrochemical Commission; 2004 
19. Diagnostic Imaging Council CT Committee. The Measurement, Reporting and Management of Radiation Dose in CT. College Park, Maryland: American Association of Physicists in Medicine; 2008. AAPM Report No. 96

20. McParland BJ. A study of patient radiation doses in interventional radiological procedures. Br J Radiol 1998;71:175-85

21. Kim S, Toncheva G, Anderson-Evans C, et al. Kerma area product method for effective dose estimation during lumbar epidural steroid injection procedures: phantom study. AJR Am J Roentgenol 2009;192:1726-30

22. Mulkens TH, Bellinck $P$, Baeyaert $M$, et al. Use of an automatic exposure control mechanism for dose optimization in multi-detector row CT examinations: clinical evaluation. Radiology 2005;237:213-23
23. Kalender WA, Buchenau S, Deak P, et al. Technical approaches to the optimisation of CT. Phys Med 2008;24:71-79. Epub 2008 Mar 10

24. Smith AB, Dillon WP, Lau BC, et al. Radiation dose reduction strategy for CT protocols: successful implementation in neuroradiology section. Radiology 2008;247:499-506

25. Flicek KT, Hara AK, Silva AC, et al. Reducing the radiation dose for CT colonography using adaptive statistical iterative reconstruction: a pilot study. AJR Am J Roentgenol 2010;195:126-31

26. Blankstein R, Bolen MA, Pale R, et al. Use of $100 \mathrm{kV}$ versus $120 \mathrm{kV}$ in cardiac dual source computed tomography: effect on radiation dose and image quality. Int J Cardiovasc Imaging 2010;27:579-86 